\title{
Limits of metastability in amorphous ices: the neutron scattering Debye-Waller factor
}

\author{
Katrin Amann-Winkel, ${ }^{* a}$ Florian Löw, ${ }^{b}$ Philip H. Handle, ${ }^{a}$ Wiebke Knoll, ${ }^{c d}$ \\ Judith Peters, ${ }^{c d e}$ Burkhard Geil, ${ }^{f}$ Franz Fujara ${ }^{b}$ and Thomas Loerting ${ }^{a}$
}

Received 10th August 2012, Accepted 19th October 2012

DOI: $10.1039 / \mathrm{c} 2 \mathrm{cp} 42797 \mathrm{~d}$

Recently, it became clear that relaxation effects in amorphous ices play a very important role that has previously been overlooked. The thermodynamic history of amorphous samples strongly affects their transition behavior. In particular, well-relaxed samples show higher thermal stability, thereby providing a larger window to investigate their glass transitions. We here present neutron scattering experiments using fixed elastic window scans on relaxed forms of amorphous ice, namely expanded high density amorphous ice (eHDA), a variant of low density amorphous ice (LDA-II) and hyperquenched glassy water (HGW). These amorphous ices are expected to be true glassy counterparts of deeply supercooled liquid water, therefore fast precursor dynamics of structural relaxation are expected to appear below the calorimetric glass transition temperature. The Debye-Waller factor shows a very weak sub- $T_{\mathrm{g}}$ anomaly in some of the samples, which might be the signature of such fast precursor dynamics. However, we cannot find this behavior consistently in all samples at all reciprocal length scales of momentum transfer.

\section{Introduction}

Water's phase diagram is very rich. Sixteen polymorphs have been isolated and characterized crystallographically so far. ${ }^{1}$ At pressures $P<200 \mathrm{MPa}$ three low-density polymorphs of ice $\left(\rho<1 \mathrm{~g} \mathrm{~cm}^{-3}\right)$ are known, namely the two polytypes hexagonal (ice Ih) and cubic ice (ice Ic) and in addition ice XI, which differs from hexagonal ice in that its sublattice of hydrogen atoms is ordered. At pressures $200 \mathrm{MPa}<P<$ $60 \mathrm{GPa}$ twelve high-density polymorphs $\left(>1 \mathrm{~g} \mathrm{~cm}^{-3}\right)$ showing seven different oxygen-ordered hydrogen-bonded networks are known, which may be either hydrogen-ordered or hydrogendisordered. Beyond $60 \mathrm{GPa}$ ice $\mathrm{X}$ dominates the phase diagram, which contains symmetric OHO-hydrogen bonds. In addition to these stable or metastable polymorphs amorphous ices are also known, which do not show long-range ordering of oxygen or hydrogen atoms. ${ }^{2}$ Also in the case of amorphous ices low- $\left(<1 \mathrm{~g} \mathrm{~cm}^{-3}\right)$ and high-density forms $\left(>1 \mathrm{~g} \mathrm{~cm}^{-3}\right)$ are

${ }^{a}$ Institute of Physical Chemistry, University of Innsbruck, Innrain 52a, 6020 Innsbruck, Austria.E-mail: katrin.winkel@uibk.ac.at

${ }^{b}$ Institut für Festkörperphysik, Technische Universität Darmstadt, Hochschulstr. 6, 64289 Darmstadt, Germany

${ }^{c}$ Institut Laue Langevin, 6 rue J. Horowitz, BP 156, 38042 Grenoble Cedex 9, France

${ }^{d}$ Universite Joseph Fourier Grenoble I, UFR PhITEM, 38041 Grenoble Cedex 9, France

${ }^{e}$ Institut de Biologie Structurale, J.-P. Ebel, UMR 5075, CNRS-CEA-UJF, 41 rue J. Horowitz, 38027 Grenoble, France

${ }^{f}$ Institut für Physikalische Chemie, Georg-August Universität Göttingen, Tammanstr. 6, 37077 Göttingen, Germany known, where the former exist metastably at $P<200 \mathrm{MPa}$ and the latter metastably at $P>200 \mathrm{MPa}^{2,3}$ For more than 25 years now, the sharp, first-order like transition between the low- and the high-density amorphous forms ${ }^{3}$ has sparked considerable scientific interest, because of the possibility that the transition line between the amorphous forms may continue at higher temperatures as a first-order phase boundary between low- (LDL) and high-density liquid (HDL) water. ${ }^{4}$

In spite of this research effort, the question of whether the amorphous forms are linked to deeply supercooled, ultraviscous liquids has not been resolved entirely to date. While some experimental data are interpreted in favour of a glass-toliquid transition and homogeneous glassy nature of the amorphous ices, ${ }^{5-12}$ other experimental data support the picture of heterogeneous nano-crystalline material showing crystal-like properties. ${ }^{13-17}$ Recent experiments contain some clues as to why experimental data on amorphous ices provide us with two diametrically opposed views. The first clue is provided by Nelmes et al., ${ }^{18}$ who demonstrate that the state of relaxation of high-density amorphous (HDA) ice can affect its properties significantly. They make a distinction between a relaxed form of HDA close to the metastable equilibrium (called expanded HDA, eHDA) and the "traditionally" studied, unrelaxed form of HDA produced by pressure-induced amorphization of hexagonal ice at $77 \mathrm{~K}$ (called unannealed HDA, uHDA). ${ }^{19}$ These two are highly similar in terms of radial distribution functions, ${ }^{2}$ but differ significantly in terms of strain and transformation kinetics. ${ }^{18,20}$ While eHDA can be regarded as 


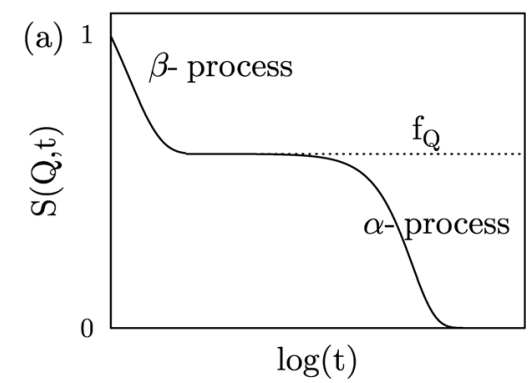

(b)

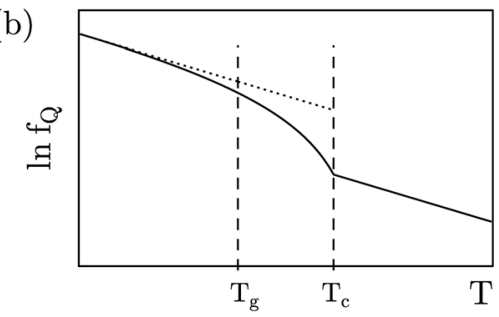

Fig. 1 (a) Sketch of the intermediate incoherent scattering function $S(Q, t)$ that decays, below $T_{\mathrm{c}}$, in two steps as predicted by modecoupling theory (MCT). (b) Sketch of the temperature dependence of the Debye-Waller factor $f_{Q}(T)$ as predicted by MCT.

being close to the metastable equilibrium, ${ }^{20}$ uHDA does not constitute any particular state of the amorphous water network. ${ }^{21}$ Recent work by Winkel et al. shows that eHDA, but not UHDA, is the one, which may co-exist with the low-density form. ${ }^{20}$ Therefore, eHDA is the best candidate to be investigated as a possible link to HDL, but not uHDA, which has been investigated in most studies in the past.

The second clue is provided by Winkel et al. ${ }^{22}$ and Elsaesser et al., ${ }^{10}$ who note that there is also a difference between the low-density amorphous (LDA) ice forms that are produced from UHDA (called LDA-I) and produced from eHDA (called LDA-II). This difference manifests itself as a subtly different intermediate range structural ordering ${ }^{22}$ and as a higher resistance of LDA-II toward crystallization to ice I. ${ }^{10}$ This is interpreted in the sense that LDA-I contains nm-sized ice I domains, which cannot be detected by common powder X-ray or neutron diffraction methods, whereas LDA-II does not contain a significant number of such ice I domains. If such nm-sized ice I domains survive the HDA $\rightarrow$ LDA transition, this would imply that these ice I domains may also be present in UHDA, but not in eHDA.

All the amorphous forms of ice discussed so far are obtained via pressure-induced amorphization of hexagonal ice at $77 \mathrm{~K}$, followed by changes in pressure and temperature. In addition to this preparation route starting from the crystalline solid, it is also possible to prepare the low-density form of amorphous ice by deposition of water vapour on cold substrates ${ }^{23}$ and by cooling of liquid water droplets at rates of $>10^{6} \mathrm{~K} \mathrm{~s}^{-1} \cdot{ }^{24-26}$ Because of these ultrahigh cooling rates the latter amorphous deposit is referred to as "hyperquenched glassy water" (HGW). Since water is a particularly bad glass-former, such high cooling rates are necessary to avoid crystallization to ice I upon cooling. HGW is obtained by vitrification of the liquid and, therefore, the most natural choice is to search for the devitrification transition (i.e., the glass-to-liquid transition) in this material.
In general, there are various experimental methods to investigate a glass-to-liquid transition in glassy materials, the most common being the measurement of isobaric heat capacity by calorimetry or the measurement of thermal expansion coefficient by dilatometry. The glass transition temperature $T_{\mathrm{g}}$ depends on the timescale of the experiment, and a glasstransition range best describes the transition from the nonequilibrium glass to the equilibrium liquid. ${ }^{27}$ For water and amorphous ice these measurements are quite challenging, since amorphous ice crystallizes to cubic ice not far above the glasstransition onset and before the glass-transition endpoint, and so, the window for measuring a possible glass-to-liquid transition is very small. Using calorimetry, the onset of the glass-to-liquid transition for $\mathrm{HGW}$ as well as LDA was determined to be at $\sim 136 \mathrm{~K}$, at a heating rate of $30 \mathrm{~K} \mathrm{~min}^{-1} \cdot 10,28$ Another less common method is inelastic neutron scattering, which gives direct access to the time dependence of correlation functions and allows to study dynamic or relaxation processes on the nanosecond time scale.

In such experiments one measures the dynamic structure or intermediate scattering function $S(Q, t)$. For supercooled liquids the mode-coupling theory $(\mathrm{MCT})^{29}$ predicts a two step decay of $S(Q, t)$ : the microscopic correlation function decays towards an intermediate plateau, which has the value of the generalized Debye-Waller factor $f_{Q}$. The long time tail of this fast decay step is called $\beta$ process. $S(Q, t)$ finally decays to zero ( $\alpha$ process), see Fig. 1a. Above the caloric glass transition temperature $T_{\mathrm{g}}$ the Debye-Waller factor $f_{Q}$ decreases more rapidly with increasing temperature up to a critical temperature $T_{\mathrm{c}}$, where another change in the temperature dependence appears. This behavior is schematically plotted in Fig. $1 b$. Ideally, the temperature dependence of the Debye-Waller factor allows to locate $T_{\mathrm{c}}$ and, therefore, this is a signature for the glass transition. Experimental results for polymers, ${ }^{30,31}$ fragile molecular glasses, ${ }^{32}$ the hydrogen network glycerol ${ }^{33}$ as well as the strong network glass former $\mathrm{B}_{2} \mathrm{O}_{3}{ }^{34}$ are in good agreement with these predictions of the mode-coupling theory.

The long-time limit of the intermediate scattering function $S(Q, t \rightarrow \infty)$, which is equivalent to the strict elastic scattering intensity $S(Q, \omega=0)=f_{Q}(T)$, can easily be measured by so-called elastic fixed window scans. ${ }^{32}$ A decrease in $f_{Q}(T)$ when heating the system is indicative of the increasing probability of inelastic scattering. Quantitatively, MCT predicts

$$
f_{Q}= \begin{cases}f_{0}(Q, T)+h(Q) \sqrt{\frac{T_{\mathrm{c}}-T}{T}} & T<T_{\mathrm{c}} \\ f_{0}(Q, T) & T>T_{\mathrm{c}}\end{cases}
$$

with $h(Q)$ being the amplitude of the precursor process at $T_{\mathrm{c}}$.

For a harmonic system this change follows the exponential function $f_{0}(Q, T)=\exp [W(Q, T)] S(Q)$, where $W$ is proportional to the temperature $W(Q, T) \propto-Q^{2} T$. Additional dynamic behavior, e.g. precursor effects below the glass transition temperature, causes a deviation from harmonic $W \propto T$ behavior to lower values. Such an experiment, which looks for an anomaly in the Debye-Waller factor according to eqn (1), has already been performed previously using the high- $Q$ backscattering spectrometer IN13 on UHDA and LDA-I. ${ }^{35,36}$ An absence of such precursor dynamics in LDA-I and UHDA is reported in this study. In these unrelaxed amorphous ices the 
critical temperature $T_{\mathrm{c}}$ is above the crystallization temperature. Hence, $T_{\mathrm{c}}$ was not accessible in this previous study. In view of the above-mentioned recent developments in our understanding of amorphous ices we report here a complementary study on LDA-II and eHDA as well as HGW with the focus on assessing possible precursor dynamics and crystallization stability of these materials.

\section{Experimental}

The neutron scattering experiments were performed using the backscattering spectrometer IN 13 at the ILL, Grenoble. The IN 13 standard setting with an incident neutron energy of $16.45 \mathrm{meV}$ and an energy resolution of $8 \mu \mathrm{eV}$ was used. The elastic intensity was sampled in a wide range of momentum transfer $0.1 \AA^{-1}<Q<4.7 \AA^{-1}$, being sensitive to short range diffusion in the high $Q$ range. The elastic intensity was measured as a function of temperature $T$ in the range of 5-180 K, using a helium closed cycle displex cryostat. The data were treated with the program $L A M P^{37}$ from the standard ILL library to apply standard data corrections (background subtraction, absorption, detector normalization). Multiple scattering corrections were not applied, which is justified by the high transmission of the samples of $91.5-94.3 \%$. To normalize the static structure factor, a vanadium standard was measured at $70 \mathrm{~K}$.

Since the amorphous ices are metastable states at ambient pressure, the samples have to be loaded at $T \leq 80 \mathrm{~K}$. Therefore, the powdered ice was filled under liquid nitrogen into a standard aluminium sample holder, with a thickness of $0.5 \mathrm{~mm}$ ( $\mathrm{D}_{2} \mathrm{O}$-samples) and $0.3 \mathrm{~mm}\left(\mathrm{H}_{2} \mathrm{O}\right.$-samples), thereby compromising between achieving good count rates and avoiding multiple scattering. The can was mounted under liquid nitrogen onto the center stick and then moved into the cryostat at $80 \mathrm{~K}$.

\subsection{Sample preparation}

The high pressure polymorphs eHDA and LDA-II were prepared in a piston cylinder apparatus using a material testing machine (Zwick, model BZ100/TL3S) to apply uniaxial pressure. The samples were prepared by amorphization of hexagonal ice at liquid nitrogen temperature, getting uHDA, directly followed by warming uHDA at $1.1 \mathrm{GPa}$ to $160 \mathrm{~K}$ to obtain very high density amorphous (VHDA) ice. ${ }^{2}$ The preparation method is described in detail elsewhere. ${ }^{38}$ In brief, eHDA is obtained by decompression of VHDA at $140 \mathrm{~K}$ or $143 \mathrm{~K}$, respectively, using deionised water $\left(\mathrm{H}_{2} \mathrm{O}\right)$ as well as fully deuterated water $\left(\mathrm{D}_{2} \mathrm{O}\right.$, euriso-top $\left.99.97 \%\right) .{ }^{39}$ To prepare eHDA the decompression process is stopped at $0.1 \mathrm{GPa}$ by immersing the sample in liquid nitrogen. LDA-II can be prepared either by decompression of the sample at $140 \mathrm{~K}$ to $\leq 6 \mathrm{MPa},{ }^{38}$ or, as done here, by heating eHDA to $140 \mathrm{~K}$ at ambient pressure. In general we discriminate low density amorphous ice which is obtained via the eHDA-state (LDA-II) or by heating UHDA (LDA-I). ${ }^{22}$

Hyperquenched glassy water (HGW) was prepared using the so-called hyperquench technique, developed by Mayer. ${ }^{25}$ Micrometer-sized water droplets are deposited on a copper plate $(T=84 \mathrm{~K})$ inside a vacuum chamber at $P \sim 6 \times$ $10^{-4}$ mbar. ${ }^{28}$ The water droplets enter the vacuum chamber through an aperture of $300 \mu \mathrm{m}$ in diameter. The technique reaches ultrafast cooling rates up to $10^{7} \mathrm{~K} \mathrm{~s}^{-1}$. The deposition time is $\sim 30 \mathrm{~min}$. Additionally the $\mathrm{HGW}$ samples were annealed inside the cryostat prior to the measurement for 90 min at $132 \mathrm{~K}\left(\mathrm{D}_{2} \mathrm{O}\right)$ or $130 \mathrm{~K}\left(\mathrm{H}_{2} \mathrm{O}\right)$, respectively, according to the procedure described by Kohl et al. ${ }^{28}$ This annealing step slightly below the caloric glass transition leads to an enthalpy relaxation of the glass.

Using fully deuterated samples we are able to monitor structural changes in the sample during heating, i.e., transformation and/or crystallization of the amorphous ice. Heavy water is a predominant coherent scatterer with a coherent cross section per atom of $\sigma_{\text {coh }}=5.118$ barn and an incoherent cross section per atom of $\sigma_{\text {inc }}=1.387$ barn. $\mathrm{H}_{2} \mathrm{O}$ instead is a predominant incoherent scatterer with $\sigma_{\text {coh }}=0.039$ barn and $\sigma_{\text {inc }}=56.04$ barn. Therefore, light water shows a nearly flat, $Q$ independent scattering.
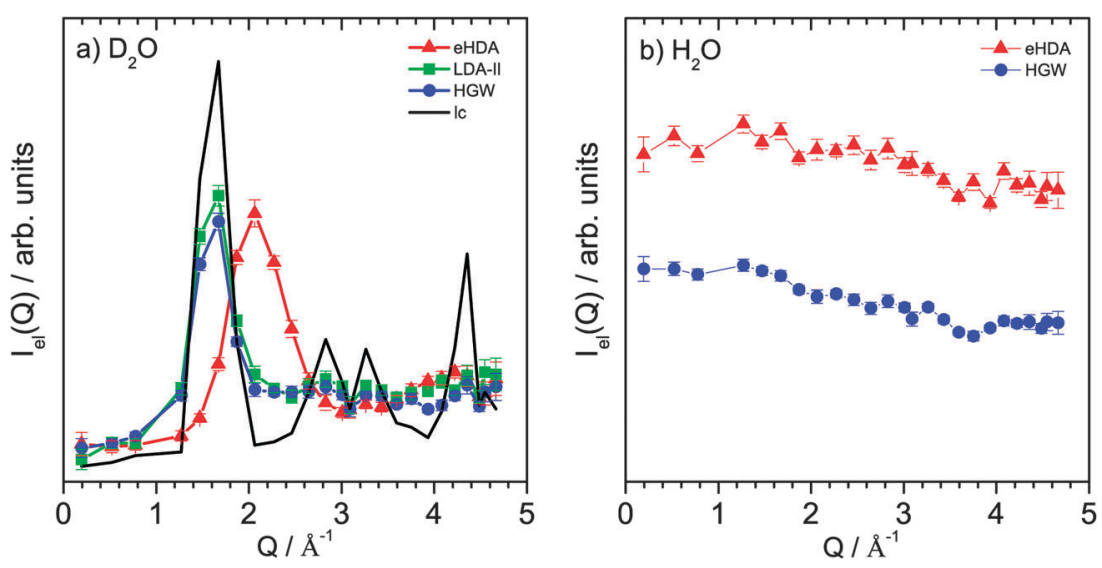

Fig. 2 Elastic intensity $I_{\mathrm{el}}(Q, \omega=0)$ of samples \#1, \#4 (eHDA, red triangles), \#1a (LDA-II, green squares) and \#2, \#3 (HGW, blue circles), measured at $T=5 \mathrm{~K}\left(\mathrm{D}_{2} \mathrm{O}\right)$ and $T=10 \mathrm{~K}\left(\mathrm{H}_{2} \mathrm{O}\right)$, respectively. The deuterated samples (a) clearly show the characteristic signatures of amorphous ices. For comparison, the elastic intensity of cubic ice is shown (black line), which has been obtained by heating sample \#la to $180 \mathrm{~K}$ and cooling back to $5 \mathrm{~K}$. Fully hydrogenated samples (b) do not show structural features. Intensities are normalized to vanadium and data are shifted for clarity. 


\begin{tabular}{|c|c|c|c|c|}
\hline & Sample & $\begin{array}{l}\text { Temperature } \\
\text { program }\end{array}$ & $\begin{array}{l}\text { Step size } \\
(\Delta T)\end{array}$ & $\begin{array}{l}\text { Transition } \\
\text { during heating }\end{array}$ \\
\hline$\overline{\# 1}$ & $\begin{array}{l}\text { eHDA } \\
\left(\mathrm{D}_{2} \mathrm{O}\right)\end{array}$ & $\underset{\rightarrow 5 \mathrm{~K} \rightarrow 137 \mathrm{~K}}{5}$ & $2-10 \mathrm{~K}$ & $\begin{array}{l}\text { Transformation } \\
\text { to LDA-II }\end{array}$ \\
\hline$\# 1 \mathrm{a}$ & LDA-II & $\begin{array}{l}5 \mathrm{~K} \rightarrow 5 \mathrm{~K} \\
\rightarrow 50 \mathrm{~K}\end{array}$ & $2-10 \mathrm{~K}$ & Crystallization \\
\hline$\# 2$ & $\mathrm{HGW}\left(\mathrm{D}_{2} \mathrm{O}\right)$ & $5 \mathrm{~K} \rightarrow 180 \mathrm{~K}$ & $2.5-15 \mathrm{~K}$ & Crystallization \\
\hline \#3 & $\mathrm{HGW}\left(\mathrm{H}_{2} \mathrm{O}\right)$ & $10 \mathrm{~K} \rightarrow 180 \mathrm{~K}$ & $2.5-15 \mathrm{~K}$ & Crystallization \\
\hline$\# 4$ & $\begin{array}{l}\text { eHDA } \\
\left(\mathrm{H}_{2} \mathrm{O}\right)\end{array}$ & $10 \mathrm{~K} \rightarrow 140 \mathrm{~K}$ & $3-20 \mathrm{~K}$ & $\begin{array}{l}\text { Transformation } \\
\text { to LDA-II }\end{array}$ \\
\hline
\end{tabular}

We performed IN13 scans at various temperatures, where the step size was $10-20 \mathrm{~K}$ at low temperatures and reduced to $2-3 \mathrm{~K}$ when approaching the caloric glass transition temperature (see Table). Due to limited beam time, the step size is bigger for sample \#4. Data accumulation time was 1 hour in all cases.

\section{Results and discussion}

\subsection{Crystallization behavior}

Fig. 2 shows the elastic intensity $I_{\mathrm{el}}(Q)=I(Q, 0 \pm \Delta E)$ of all samples measured at low temperatures, i.e., $T=5 \mathrm{~K}$ for the $\mathrm{D}_{2} \mathrm{O}$ samples and $T=10 \mathrm{~K}$ for the $\mathrm{H}_{2} \mathrm{O}$ samples, respectively. The measured intensities are normalized to vanadium. The deuterated samples (Fig. 2a) show the characteristic broad halo peak, as expected for amorphous ice. This prominent feature is known to correlate with the intermediate range structural organization in glasses and liquids. ${ }^{22}$ The first diffraction peak for eHDA (red) appears at $Q=2.1 \pm 0.1 \AA^{-1},{ }^{18}$ for LDA-II (green) and HGW (blue) at $Q=1.7 \pm 0.1 \AA^{-1} \cdot{ }^{40}$ This corresponds to a $d$-spacing of $3.0 \AA$ and $3.7 \AA$, respectively. The black line shows the elastic intensity of cubic ice, which is obtained after warming LDA-II to $180 \mathrm{~K}$. In cubic ice Bragg reflections appear at $Q=$ 1.7, 2.8, 3.3, $4.3 \AA^{-1}$. Due to their predominant incoherent scattering, the fully hydrogenated samples (Fig. 2b) do not show structural features.

The temperature scan of sample \#1 (eHDA, $\left.\mathrm{D}_{2} \mathrm{O}\right)$ for different $Q$ values is shown in Fig. 3a. During heating eHDA transforms to LDA-II at $\approx 123 \mathrm{~K}$. This transformation is indicated by an increase in the elastic intensity at $Q=1.7 \AA^{-1}$ (black squares) and a decrease in the signal at $Q=2.1 \AA^{-1}$ (open diamonds). This directly reflects the increase in the maximum of the LDA structure factor and the corresponding loss of the HDA structure factor. At $Q=4.5 \AA^{-1}$ (filled circles) the structure factors of eHDA and LDA-II are very similar, as can be seen in Fig. 2a. Hence, the transformation from eHDA to LDA-II cannot be monitored at this $Q$-value. The measurements presented here show that the eHDA $\rightarrow$ LDA-II transition takes place at $123 \mathrm{~K}$ and is, therefore, $\approx 20 \mathrm{~K}$ higher compared to previous measurements on uHDA. ${ }^{36}$ This increase in thermal stability is consistent with neutron scattering measurements by Nelmes et al. ${ }^{18}$ and calorimetric measurements by Winkel et al. ${ }^{20}$

After transition to LDA-II, the sample was cooled back to $5 \mathrm{~K}$ and again a temperature scan was performed (Fig. 3b). The increase in the elastic intensity at $Q=1.7 \AA^{-1}$ (black squares) and the decrease at $Q=2.3 \AA^{-1}$ (open triangles) clearly mark crystallization of the sample. Here, the increase is due to Bragg reflection of cubic ice with the corresponding loss of LDA. We observe a similar behavior for sample \#2
( $\mathrm{HGW}, \mathrm{D}_{2} \mathrm{O}$ ), as shown in Fig. 3c. Since the two low density amorphous ices LDA-II and HGW have the same density and structure to within the limits of this measurement, we monitor the evolution of the elastic intensity with increasing temperature at the same $Q$-values, namely $Q=1.7,2.3,4.5 \AA^{-1}$. The HGW $\rightarrow$ cubic ice transition takes place at $139 \mathrm{~K}$. The LDA-II $\rightarrow$ cubic ice transition instead takes place at $149 \mathrm{~K}$. That is, the crystallization temperature of LDA-II is nearly $10 \mathrm{~K}$ above that of HGW. Previous measurements on LDA-I, also done using IN13 at a similar heating rate, ${ }^{35}$ showed that LDA-I crystallizes at $135 \mathrm{~K}$.

A complementary study on the transition behavior of eHDA, LDA-II and HGW can be found in a second part of this publication. ${ }^{41}$ There we show ${ }^{2} \mathrm{H}-\mathrm{NMR}$ measurements, monitoring the spin lattice relaxation time as a parameter for the eHDA $\rightarrow$ LDA-II transition and crystallization of the latter as well as crystallization of HGW. The results of the NMR study are in total
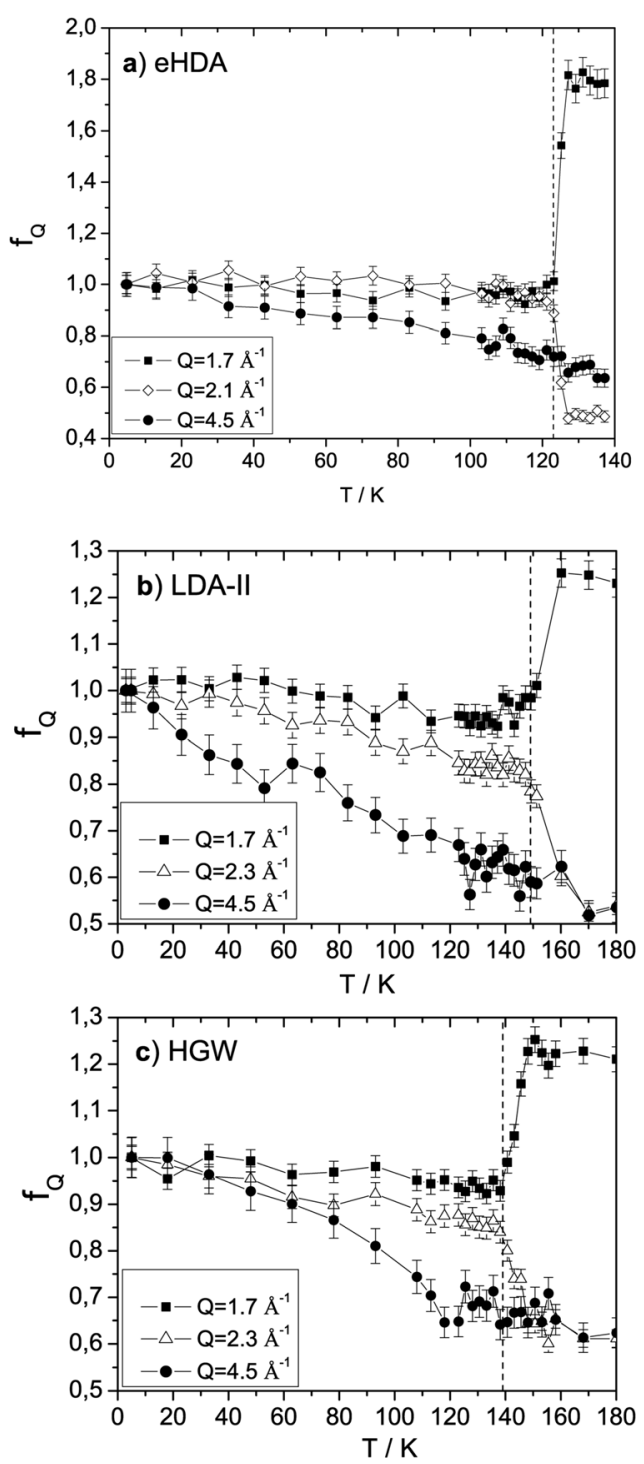

Fig. 3 Temperature dependence of the Debye-Waller factor $f_{Q}=$ $I_{\mathrm{el}}(Q, T) / I_{\mathrm{el}}(Q, 5 \mathrm{~K})$ at different $Q$-values, normalized to the elastic intensity at $5 \mathrm{~K}$, of the deuterated samples (a) \#1 eHDA, (b) \#1a LDA-II, (c) \#2 HGW. 
agreement with the results presented here obtained by neutron scattering.

\subsection{Search for precursor dynamics below the glass transition temperature}

As shown in the previous section, the relaxed amorphous ices eHDA and LDA-II now provide a $20 \mathrm{~K}$ and, respectively, $14 \mathrm{~K}$ wider window for our measurements, compared to previous studies on UHDA and LDA-I. ${ }^{36}$ This is very important, since the glass transition range overlaps with the transition temperatures. ${ }^{10,11}$ In Fig. 4 the evolution of the elastic intensity while heating is shown for all samples. Compared to Fig. 3, we now present the logarithm of the normalized intensities as a function of temperature. The logarithmic depiction is chosen since $f_{Q}(T)$ for a harmonic system follows an exponential behavior (see Introduction). In non-harmonic systems like glasses, precursor dynamics below the glass transition temperature results in an additional decrease in the elastic intensity.

The left column of Fig. 4 shows data of the high density amorphous ice samples $(\# 1, \# 4)$ up to $T=123 \mathrm{~K}$, where the sample transforms to LDA-II. The low density ices LDA-II (\#1a) (right column) and HGW (\#2, \#3) (middle column) are plotted in the temperature range up to the temperature where crystallization sets in (149 K for LDA-II and $139 \mathrm{~K}$ for HGW). The data are shown for three different $Q$ values. Assuming that the Debye model is valid, the intensity decrease can be fitted by a straight line far below $T_{\mathrm{g}}$. Here, all data are fitted in the range of $\sim 20 \mathrm{~K}$ to $\sim 100 \mathrm{~K}$ as indicated by the black lines. There are slight indications for a small deviation from the harmonic behavior at temperatures above $100 \mathrm{~K}$. If the precursor dynamics are affected by motions of very small amplitudes these deviations should become most pronounced at high $Q$ values. This could explain why the possible effect in Fig. 4 is only visible for $Q \geq 3.9 \AA^{-1}$.

In order to average out statistical fluctuations of intensity, we summed up the elastic intensities for all $Q$ values in the range of $1.7 \leq Q \leq 4.5 \AA^{-1}$. As mentioned in the Introduction, the Debye Waller factor is proportional to the product of temperature and $Q^{2}$. Therefore, we first divided the logarithm of the normalized intensity by $Q^{2}$ before summing up (see Fig. 5). The data are fitted linearly in the range of $\sim 20 \mathrm{~K}$ to $\sim 100 \mathrm{~K}$ (eHDA samples) and $\sim 110 \mathrm{~K}$ (HGW, LDA-II samples) as indicated by
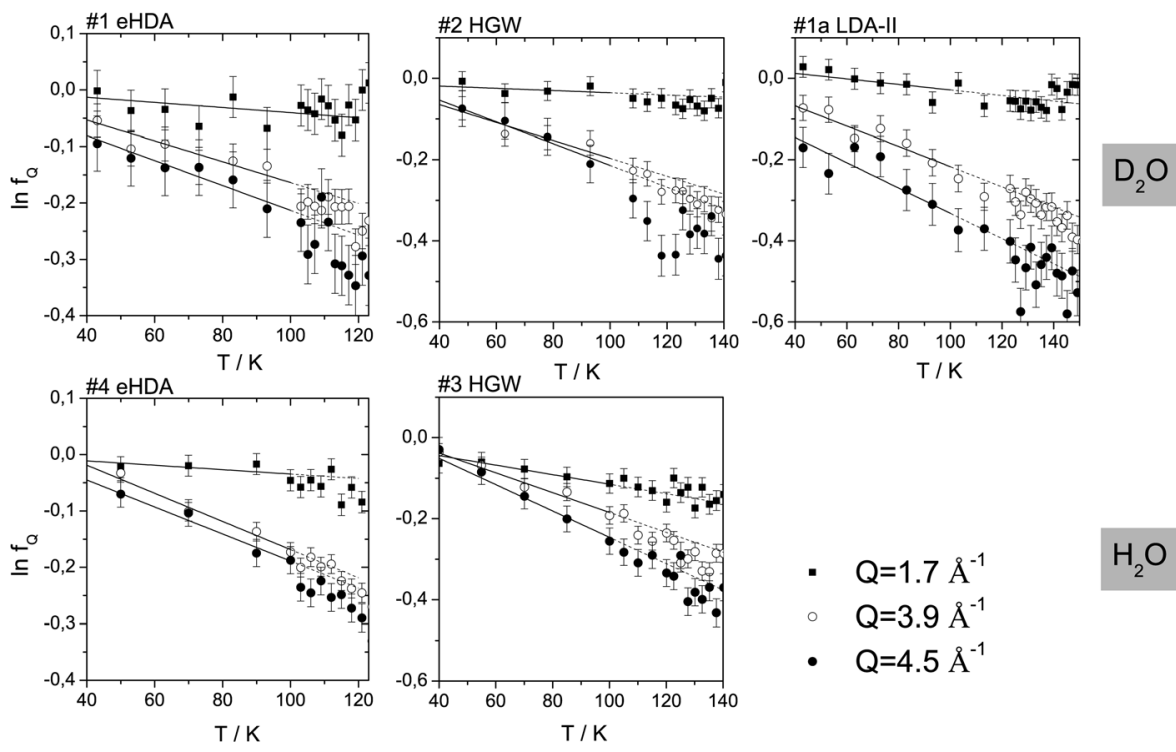

Fig. 4 Temperature dependence of the elastic intensity at $Q=1.7,3.9,4.5 \AA^{-1}$, normalized to the elastic intensity at low temperature and logarithmized. The top row shows the deuterated samples \#1 (eHDA), \#2 (HGW), \#1a (LDA-II), the bottom row shows the hydrogenated samples $\# 4$ (eHDA) and \#3 (HGW). LDA-II has only been measured as a deuterated sample.
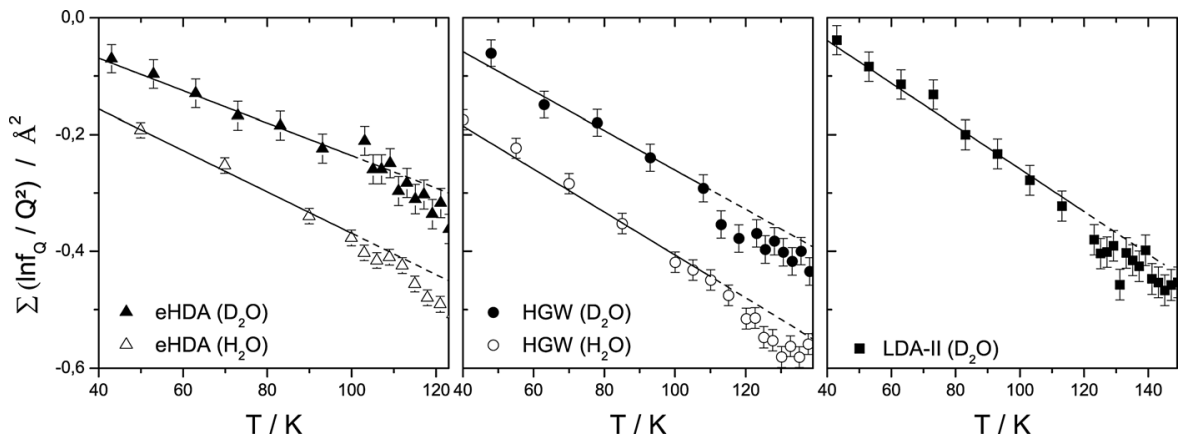

Fig. 5 The logarithm of the normalized intensities divided by $Q^{2}$ and then summed up in the range of $1.7 \leq Q \leq 4.5 \AA^{-1}$. The deuterated samples are shown as open symbols and the hydrogenated samples as open symbols. The hydrogenated data are shifted by $-0.1 \AA^{2}$ for clarity. LDA-II was measured as a deuterated sample only. 
the black lines. The linear fit is further extrapolated to higher $T$ (dotted line). In this generalized plot a small anomaly similar to the one shown in Fig. $1 \mathrm{~b}$ is visible in the deuterated as well as in the hydrogenated sample, but it is hard to show statistical significance. The weak deviation from linearity is slightly more pronounced for the hydrogenated samples. By comparing the upper limits of the error bars with the extrapolated linearity, we can infer more significant deviations at $>110 \mathrm{~K}$ for the hydrogenated eHDA sample and at $>120 \mathrm{~K}$ for the hydrogenated HGW sample. In both cases this is the temperature range in which we would expect precursor dynamics, judging from the calorimetry measurements which indicate the onset of $T_{\mathrm{g}}$ to be at $115 \mathrm{~K}$ for $\mathrm{eHDA}^{11}$ and $136 \mathrm{~K}$ for $\mathrm{HGW} .{ }^{28}$ However, in the case of deuterated samples the upper limit of the error bars is only partly below the extrapolated low-temperature linearity, even though all data points at $T>$ $110 \mathrm{~K}$ in the case of eHDA and HGW fall below the extrapolated line. That is, sub- $T_{\mathrm{g}}$ precursor dynamics may underlie the data, but cannot be inferred with sufficient significance from the data set.

\section{Conclusions}

The transformation behavior of eHDA, monitored by the intensity change with temperature at $Q=1.7,2.1 \AA^{-1}$ clearly shows that the transition to LDA-II appears at $123 \mathrm{~K}$, which is about $20 \mathrm{~K}$ higher than the transition from uHDA to LDA-I. This confirms earlier measurements on eHDA with different techniques. $^{18,20}$

A novel finding is that the so produced LDA-II shows a crystallization temperature of $149 \mathrm{~K}$, whereas the crystallization of $\mathrm{HGW}$ already starts at $139 \mathrm{~K}$, i.e., $10 \mathrm{~K}$ lower. LDA-I even crystallizes $14 \mathrm{~K}$ lower than LDA-II when heating the system in steps of $2 \mathrm{~K}$ every few hours. ${ }^{35}$ That is, LDA-II is more stable against crystallization than LDA-I. This result is also confirmed by NMR measurements presented in a second part of this publication. ${ }^{41}$ Enhanced stability of LDA-II towards crystallization was previously demonstrated by Elsaesser et al. using X-ray diffraction and calorimetric measurements. ${ }^{10}$ In their study they used LDA-II, which was produced by full decompression of VHDA at $140 \mathrm{~K}$ to $6 \mathrm{MPa}$. Also due to faster heating rates, they observed a difference in thermal stability of only $5 \mathrm{~K}$. Neutron scattering measurements combined with empirical potential structure refinement (EPSR) showed that LDA-II (produced by decompression of VHDA) is more relaxed compared to LDA-I (produced by heating uHDA). ${ }^{22}$ That is, comparing the present study with literature data ${ }^{10,22}$ and NMR-data ${ }^{41}$ it shows that LDA-II can be produced both by decompression of VHDA at $140 \mathrm{~K}$ and by heating eHDA at ambient pressure.

A very weak sub- $T_{\mathrm{g}}$ anomaly, suggesting possible precursor dynamics below the caloric glass transition onset, can be identified in some of the samples. While these deviations are slightly visible in the hydrogenated samples (Fig. 5), they cannot be corroborated with certainty from the data obtained from deuterated samples.

\section{Acknowledgements}

We are grateful for financial support from the Austrian Science Fund (Hertha-Firnberg award T463 to K. A.-W. and START award Y391 to T.L.) and the European Research Council (ERC Starting Grant SULIWA to T.L.).

\section{References}

1 C. G. Salzmann, P. G. Radaelli, B. Slater and J. L. Finney, Phys. Chem. Chem. Phys., 2011, 13, 18468.

2 T. Loerting, K. Winkel, M. Seidl, M. Bauer, C. Mitterdorfer, P. H. Handle, C. G. Salzmann, E. Mayer, J. L. Finney and D. T. Bowron, Phys. Chem. Chem. Phys., 2011, 13, 8783.

3 O. Mishima, L. D. Calvert and E. Whalley, Nature, 1985, 314, 76.

4 P. H. Poole, F. Sciortino, U. Essmann and H. E. Stanley, Nature, 1992, 360, 324.

5 G. Johari, A. Hallbrucker and E. Mayer, Nature, 1987, 330, 552.

6 G. Johari, J. Phys. Chem. B, 1998, 102, 4711.

7 R. S. Smith and B. D. Kay, Nature, 1999, 398, 788.

8 O. Mishima and Y. Suzuki, J. Chem. Phys., 2001, 115, 4199.

9 O. Mishima, J. Chem. Phys., 2004, 121, 3161.

10 M. S. Elsaesser, K. Winkel, E. Mayer and T. Loerting, Phys. Chem. Chem. Phys., 2010, 12, 708.

11 M. Seidl, M. S. Elsaesser, K. Winkel, G. Zifferer, E. Mayer and T. Loerting, Phys. Rev. B: Condens. Matter Mater. Phys., 2011, 83, 100201 .

12 O. Andersson, Proc. Natl. Acad. Sci. U. S. A., 2011, 108, 11013.

13 J. S. Tse, D. D. Klug, C. A. Tulk, I. Swainson, E. C. Svensson, C.-K. Loong, V. Shpakov, V. R. Belosludov, R. V. Belosludov and Y. Kawazoe, Nature, 1999, 400, 647.

14 J. S. Tse, D. D. Klug, C. A. Tulk, E. C. Svensson, I. Swainson, V. P. Shpakov and V. R. Belosludov, Phys. Rev. Lett., 2000, 85, 3185.

15 H. Schober, M. M. Koza, A. Tolle, C. Masciovecchio, F. Sette and F. Fujara, Phys. Rev. Lett., 2000, 85, 4100.

16 O. Andersson and H. Suga, Phys. Rev. B: Condens. Matter Mater. Phys., 2002, 65, 140201.

17 M. M. Koza, H. Schober, B. Geil, M. Lorenzen and H. Requardt, Phys. Rev. B: Condens. Matter Mater. Phys., 2004, 69, 024204.

18 R. J. Nelmes, J. S. Loveday, T. Strassle, C. L. Bull, M. Guthrie, G. Hamel and S. Klotz, Nat. Phys., 2006, 2, 414.

19 O. Mishima, L. D. Calvert and E. Whalley, Nature, 1984, 310, 393.

20 K. Winkel, E. Mayer and T. Loerting, J. Phys. Chem. B, 2011, 115, 14141.

21 M. M. Koza, T. Hansen, R. P. May and H. Schober, J. Non-Cryst. Solids, 2006, 352, 4988

22 K. Winkel, D. T. Bowron, T. Loerting, E. Mayer and J. L. Finney, J. Chem. Phys., 2009, 130, 204502.

23 E. F. Burton and W. F. Oliver, Proc. R. Soc. London, Ser. A, 1936, 153, 166.

24 P. Brueggeller and E. Mayer, Nature, 1980, 288, 569.

25 E. Mayer, J. Appl. Phys., 1985, 58, 663.

26 B. Mate, Y. Rodriguez-Lazcano and V. J. Herrero, Phys. Chem. Chem. Phys., 2012, 14, 10595.

27 C. A. Angell, Science, 2008, 319, 582.

28 I. Kohl, L. Bachmann, A. Hallbrucker, E. Mayer and T. Loerting, Phys. Chem. Chem. Phys., 2005, 7, 3210

29 W. Götze and L. Sjögren, Chem. Phys., 1996, 212, 47.

30 D. Richter, B. Frick and B. Farago, Phys. Rev. Lett., 1988, 61, 2465.

31 B. Frick and D. Richter, Science, 1995, 267, 1939.

32 F. Fujara and W. Petry, Europhys. Lett., 1987, 4, 921.

33 F. Fujara, W. Petry, R. M. Diehl, W. Schnauss and H. Sillescu, Europhys. Lett., 1991, 14, 563.

34 D. Engberg, L. Boerjesson, J. Swenson, L. M. Torell, W. S. Howells and A. Wannberg, Europhys. Lett., 1999, 47, 213.

35 B. Geil, M. Koza, F. Fujara, H. Schober and F. Natali, Phys. Chem. Chem. Phys., 2004, 6, 677.

36 M. Koza, B. Geil, H. Schober and F. Natali, Phys. Chem. Chem. Phys., 2005, 7, 1423.

37 D. Richard, M. Ferrand and G. J. Kearley, provided by the ILL, see www.ill.eu.

38 K. Winkel, M. Elsaesser, E. Mayer and T. Loerting, J. Chem. Phys., 2008, 128, 044510.

39 K. Winkel, et al., J. Phys.: Condens. Matter, 2008, 20, 494212.

40 D. T. Bowron, J. L. Finney, A. Hallbrucker, I. Kohl, T. Loerting, E. Mayer and A. K. Soper, J. Chem. Phys., 2006, 125, 194502.

41 F. Löw, K. Amann-Winkel, B. Geil, T. Loerting, C. Wittich and F. Fujara, Phys. Chem. Chem. Phys., 2012, submitted. 